\title{
Comparison between Continuous Regular Insulin Infusion and Single Dose Subcutaneous Long Acting Insulin Injection in Intensive Care Unit
}

\author{
Khaled Mohammed Hassan, Kareem Khaled Elhossini*, Abd Elhady Ahmed Helmy Abd Elhady
}

Department of Anesthesia, Intensive Care and Pain Management Faculty of Medicine, Sohag University, Sohag, Egypt

*Corresponding Authors: Kareem K. Elhossini, Mobile: +201064779570, Email: kareemelkhayat92@gmail.com

\begin{abstract}
Background: Stress-induced hyperglycemia is a common metabolic disorder in Intensive Care Unit (ICU) cases $(30-60 \%)$ in diabetic and non-diabetic patients. It is related to increased rates of mortality and morbidity.

Objective: The aim of the current work was to compare between glycemic control in single dose long-acting subcutaneous insulin glargine injection and standard continuous regular insulin infusion in the same critical case.

Patients and methods: This prospective clinical study period was 72 hours for every patient and conducted on 111 patients aged 20-70 years with targeted blood glucose (BG) level ranging from 100 to $200 \mathrm{mg} / \mathrm{dl}$ using a different calculated dose of standard insulin infusion according to conventional sliding scale for the 1 st $24 \mathrm{hr}$ of the study before conversion into insulin glargine single dose injection with 1-hour washout transition period.

Results: APACHE II score was significantly higher in "Uncontrolled hyperglycemia" group in comparison to "Uncontrolled hypoglycemia" group $(\mathrm{P}=0.030)$ but insignificantly different between "Controlled" group and "Uncontrolled hyperglycemia" group and between "Controlled" group and "Uncontrolled hypoglycemia" group. Daily units of insulin and mean BG level at 1st day were significantly lower in "Uncontrolled hypoglycemia" group in comparison to "Controlled" group ( $\mathrm{P}=0.002$ and 0.002 respectively) and "Uncontrolled hyperglycemia" group $(\mathrm{P}=0.004$ and 0.006 respectively) but insignificantly different between "Controlled" group and "Uncontrolled hyperglycemia" group. While mean BG level was insignificantly different among the three groups at 2nd day, it was insignificantly different between "Controlled" group and "Uncontrolled hyperglycemia" group at 3rd day.

Conclusion: It could be concluded that the use of $100 \%$ conversion single dose of long-acting insulin glargine for control of hyperglycemia in critical ICU patients can be considered as an accepted good alternative to the classic use of continuous regular insulin infusion.
\end{abstract}

Keywords: Hyperglycemia, Single-Dose Subcutaneous Long-Acting Insulin, Continuous Regular Insulin Infusion, Intensive Care Unit.

\section{INTRODUCTION}

Stress-induced hyperglycemia is a common metabolic disorder in Intensive Care Unit (ICU) cases. It is related to increased rates of mortality and morbidity $(\mathbf{1}, 2)$. In various critical conditions, the frequency of glycemic issue ranges from $30 \%$ to $60 \%$, depending on the diagnostic criteria ${ }^{(3,4)}$.

Two of the most prominent pathophysiologies of stress-induced hyperglycemia are the change in gluconeogenesis and insulin receptor sensitivity ${ }^{(5)}$. Hyperglycemia can result from several ICU care techniques, including catecholamine infusion, renal replacement therapy, concentrated glucose intravenous fluid, and various medicines ${ }^{(3)}$.

In typical ICU practice, glycemic control is frequently advised for critically cases. According to a recent guidelines, all cases that exhibit blood glucose (BG) level $>200 \mathrm{mg} / \mathrm{dl}$ due to acute hyperglycemia after ICU hospitalization should have a level of BG ranging from 140 to $200 \mathrm{mg} / \mathrm{dl}{ }^{(6)}$. In the ICU, the standard management regimen, which includes continuous regular insulin infusion, has shown to be the most effective strategy for $\mathrm{BG}$ control ${ }^{(7,8)}$.

Insulin glargine is insulin analogue which have a long-acting, "peakless" properties. It was first used in clinical practice for $\mathrm{BG}$ management in outpatient settings some years ago ${ }^{(9)}$. This form of insulin just needs a single subcutaneous (SC) injection each day, providing convenience and requiring less equipment. Several trials have demonstrated that using insulin glargine in patients with either type 1 diabetes mellitus (DM) or type 2 diabetes mellitus (T2DM) resulted in optimum BG management without hypoglycemia consequences $(\mathbf{1 0}, \mathbf{1 1})$. However, the number of trials evaluating insulin glargine BG management in the ICU context is limited.

The aim of this study was to assess glycemic control between a typical continuous infusion of regular insulin and a single-dose SC injection of insulin glargine in the same patient. We studied whether single-dose SC insulin glargine injection is inferior or not to the regular continuous insulin infusions in controlling BG in critical cases.

\section{PATIENTS AND METHODS}

This prospective clinical study included a total of 111 patients aged 20-70 years, attending at intensive care unit (ICU) at Sohag University Hospital. This study targeted blood glucose (BG) level ranging from 100 to $200 \mathrm{mg} / \mathrm{dl}$ using different doses of standard insulin infusion according to the conventional sliding scale for 
1 st $24 \mathrm{hr}$ of the study before conversion into single-dose insulin glargine.

\section{Ethical considerations:}

An approval of the study was obtained from Sohag University Academic and Ethical Committee. Every patient signed an informed written consent for acceptance of the operation. This work has been carried out in accordance with The Code of Ethics of the World Medical Association (Declaration of Helsinki) for studies involving humans.

Exclusion criteria: Patient refusal or relative's refusal in case of unconscious patients, pregnant patients, hemodynamically unstable patients (mean arterial blood pressure $<65 \mathrm{mmHg}$ ), patients who developed complications of acute hyperglycemia such as nonketotic hyperosmolar coma, diabetic ketoacidosis, and patients who have endocrinal diseases that may interfere with the result, for example, Cushing syndrome, uncompensated liver cell failure or thyrotoxicosis.

The study period was 72 hours for every one of the included 111 patients and were controlled by regular insulin infusion in the $1^{\text {st }} 24$ hours of the study according to conventional sliding scale. Then, following a 1-hour washout time, transitioned to single-dose insulin glargine subcutaneous (SC) injection. The dose of insulin glargine was equivalent to the accumulative dosage of regular insulin for every patient on the previous 24-h infusion of regular insulin (Humulin ${ }^{\circledR} \mathrm{R}$ U-100, Eli Lilly and company). The site of injection of insulin glargine (Lantus ${ }^{\circledR}$, Sanofi-Aventis, USA) was the periumbilical area and was done by ICU stuff for 2 consecutive days. Regular measurement of BG was done every $2 \mathrm{~h}$ for $72 \mathrm{hr}$ by an FIA BioMed Salut blood glucometer device. To maintain the level of glucose constant during the study period, the amount of average caloric enteral or parenteral supplement was maintained at $30 \mathrm{kcal} / \mathrm{kg}$ for all patients.

The patients were classified as "controlled group" if their BG levels were ranged from 100 to $200 \mathrm{mg} / \mathrm{dl}$ throughout the trial. Patients with BG levels $>200 \mathrm{mg} / \mathrm{dl}$ who failed blood glucose control were turned back to regular insulin continuous infusions and are classified as "uncontrolled hyperglycemia group." Patients with severe hypoglycemia (BG levels less than $100 \mathrm{mg} / \mathrm{dl}$ ) received $100 \mathrm{ml}$ of $25 \%$ intravenous (IV) dextrose solution right away, and an IV continuous infusion of dextrose solution by a syringe pump was started with measurement of BG every hour until the level stabilized. These instances were classified as members of the "uncontrolled hypoglycemia group".

The accumulative dosage of regular insulin in the $1^{\text {st }} 24 \mathrm{~h}$ of the study period was also calculated. During the 72-hour study period, blood glucose levels were measured every 2 hours. Also, the levels of glycemic control by insulin glargine were reported, both controlled and uncontrolled.
The following data have been collected from all patients:

*Demographic data: Name, age, and sex.

*Medical history: History of corticosteroid

use, and acute Physiology and Chronic Health

Evaluation (APACHE) II score

*Investigations: Blood glucose level.

The primary outcome was the percent of patients with controlled glucose level. The secondary outcomes were the factors that may affect the control of glucose level (e.g. age, history or present corticosteroids use etc).

\section{Sample size calculation:}

The sample size calculation was done by PS: Power and Sample Size Calculation software Version 3.1.2 (Vanderbilt University, Nashville, Tennessee, USA). Expected percent of patients with controlled glucose level is $70 \%$ according to a previous study ${ }^{(\mathbf{1 2})}$ with $9 \%$ precision, $80 \%$ power of the study and $95 \%$ confidence limit. Therefore, at least 100 patients are needed, and we recruited more cases to overcome dropout. Therefore, we recruited 111 patients.

\section{Statistical analysis}

SPSS v25 (IBM, Chicago, IL, USA) was used for statistical analysis. The ANOVA (F) test was used to evaluate quantitative parametric data reported as mean and standard deviation (SD). The normality of the data distribution was analyzed with the Shapiro-Wilks test and histograms. The median and interquartile range (IQR) were used to provide quantitative non-parametric data, which were then examined using the Mann Whitney-test. When applicable, qualitative data was provided as a number and percent and compared using the Fisher's Exact or chi-square $\left(\mathrm{X}^{2}\right)$ test. Statistical significance was defined as a two-tailed $\mathrm{P}$ value of less than 0.05 .

\section{RESULTS}

In this study, 138 patients were assessed for eligibility, 24 patients did not meet the criteria and 3 patients refused to participate. So, the remaining 111 enrolled participates were controlled by regular insulin infusion in the 1 st 24 hours of the study according to conventional sliding scale. Then, following a 1-hour washout time, transitioned to single-dose insulin glargine subcutaneous injection. All 111 patients were followed-up and analyzed statistically.

The age of the patients ranged from 20-68 years with a mean value of $45.45 \pm 13.01$ years. There were $84(75.7 \%)$ male patients and 27 (24.3\%) female patients. There were $16(14.4 \%)$ patients with a history of corticosteroids. APACHE score ranged from 0-30 with a median value of 6 (Table 1). 
Table (1): Patients' characteristics and APACHE II score of all studied patients

\begin{tabular}{|c|c|c|}
\hline \multicolumn{2}{|c|}{} & $\begin{array}{c}\text { Patients } \\
(\mathbf{n = 1 1 1})\end{array}$ \\
\hline \multirow{2}{*}{$\begin{array}{c}\text { Age } \\
\text { (years) }\end{array}$} & $\begin{array}{c}\text { Mean } \pm \\
\text { SD }\end{array}$ & $\begin{array}{c}45.45 \pm \\
13.01\end{array}$ \\
\cline { 2 - 3 } Sex & Range & $20-68$ \\
\hline \multirow{2}{*}{$\begin{array}{c}\text { Male } \\
\text { History of } \\
\text { corticosteroid use }\end{array}$} & Female & $27(24.7 \%)$ \\
\cline { 2 - 3 } $\begin{array}{c}\text { YPACHE II score } \\
\text { APAC }\end{array}$ & $16(14.4 \%)$ \\
\hline \multirow{2}{*}{\begin{tabular}{c} 
Nodian \\
\cline { 2 - 3 }
\end{tabular}} & Range & $05(85.6 \%)$ \\
\cline { 2 - 3 } & \multicolumn{2}{|c}{6} \\
\hline
\end{tabular}

Mean blood glucose level was significantly decreased at both 2 nd and 3rd day as compared to the 1st day (P1 <0.001 and P2 <0.001) and was insignificantly different between 2 nd day and 3rd day $(\mathrm{P} 3=0.055)$ (Table 2).
Table (2): Mean blood glucose level of all studied patients

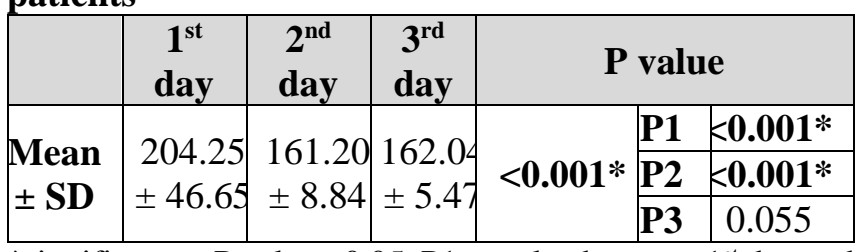

*significant as $\mathrm{P}$ value $<0.05, \mathrm{P} 1: \mathrm{p}$ value between $1^{\text {st }}$ day and $2^{\text {nd }}$ day, P2: $p$ value between $1^{\text {st }}$ day and $3^{\text {rd }}$ day, P3: $p$ value between $2^{\text {nd }}$ day and $3^{\text {rd }}$ day.

The age of the patients and sex were insignificantly different among groups $(\mathrm{P}=0.25$ and 0.906 respectively). History of corticosteroid use was significantly increased in the uncontrolled hyperglycemia group $(\mathrm{P}=0.031)$. APACHE II score was significantly higher in the "Uncontrolled hyperglycemia" group in comparison to the "Uncontrolled hypoglycemia" group $(\mathrm{P}=0.030)$ but insignificantly different between the "Controlled" group and "Uncontrolled hyperglycemia" group and between "Uncontrolled hypoglycemia" group and "Controlled" group (Table 3).

Table (3): Patients' characteristics of all groups

\begin{tabular}{|c|c|c|c|c|c|c|}
\hline \\
\hline 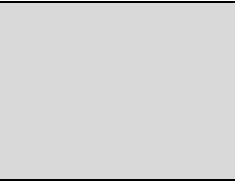 & & $\begin{array}{c}\text { Controlled } \\
(\mathrm{n}=72)\end{array}$ & $\begin{array}{l}\text { Uncontrolled } \\
\text { hyperglycemia } \\
\quad(n=28)\end{array}$ & $\begin{array}{c}\text { Uncontrolled } \\
\text { hypoglycemi } \\
\text { a } \\
(\mathbf{n}=\mathbf{1 1})\end{array}$ & $\begin{array}{c}P \\
\text { value }\end{array}$ & $\begin{array}{c}\text { Post Hoc } \\
\text { test }\end{array}$ \\
\hline $\begin{array}{c}\text { Age } \\
\text { (years) }\end{array}$ & $\begin{array}{c}\text { Mean } \pm \\
\text { SD }\end{array}$ & $\begin{array}{c}45.84 \pm \\
14.17\end{array}$ & $46.82 \pm 11.08$ & $39.36 \pm 7.41$ & 0.25 & --- \\
\hline \multirow{2}{*}{ Sex } & Male & $54(75.0 \%)$ & $22(78.6 \%)$ & $8(72.7 \%)$ & \multirow{2}{*}{0.906} & \multirow{2}{*}{---} \\
\hline & Female & $18(25.0 \%)$ & $6(21.4 \%)$ & $3(27.3 \%)$ & & \\
\hline \multirow{2}{*}{\begin{tabular}{|c|} 
History of \\
corticosteroid \\
use \\
\end{tabular}} & Yes & $15(20.8 \%)$ & $1(3.6 \%)$ & $0(0 \%)$ & \multirow{2}{*}{$0.031 *$} & \multirow[b]{2}{*}{---} \\
\hline & No & $57(79.2 \%)$ & $27(96.4 \%)$ & $11(100 \%)$ & & \\
\hline $\begin{array}{l}\text { APACHE II } \\
\text { score }\end{array}$ & Median & 6 & 8 & 4 & $0.037 *$ & $\begin{array}{l}\mathbf{P} 1=0.287 \\
\mathbf{P} 2=0.177 \\
\mathbf{P 3}=\mathbf{0 . 0 3 0} *\end{array}$ \\
\hline
\end{tabular}

*significant as P value $<0.05, \mathrm{P} 1$ : P value between "Controlled" group and "Uncontrolled hyperglycemia" group, P2: P value between "Controlled" group and "Uncontrolled hypoglycemia" group, P3: P value between "Uncontrolled hyperglycemia" group and "Uncontrolled hypoglycemia" group.

The daily units of insulin and mean blood glucose (BG) level at 1st and 2nd days were significantly lower in "Uncontrolled hypoglycemia" group in comparison to "Controlled" group and "Uncontrolled hyperglycemia" group but insignificantly different between "Controlled" group and "Uncontrolled hyperglycemia" group. The mean BG level at 3rd day was insignificantly different between "Controlled" group and "Uncontrolled hyperglycemia" group (Table 4). 
Table (4): Daily units of insulin and mean blood glucose level of all studied groups

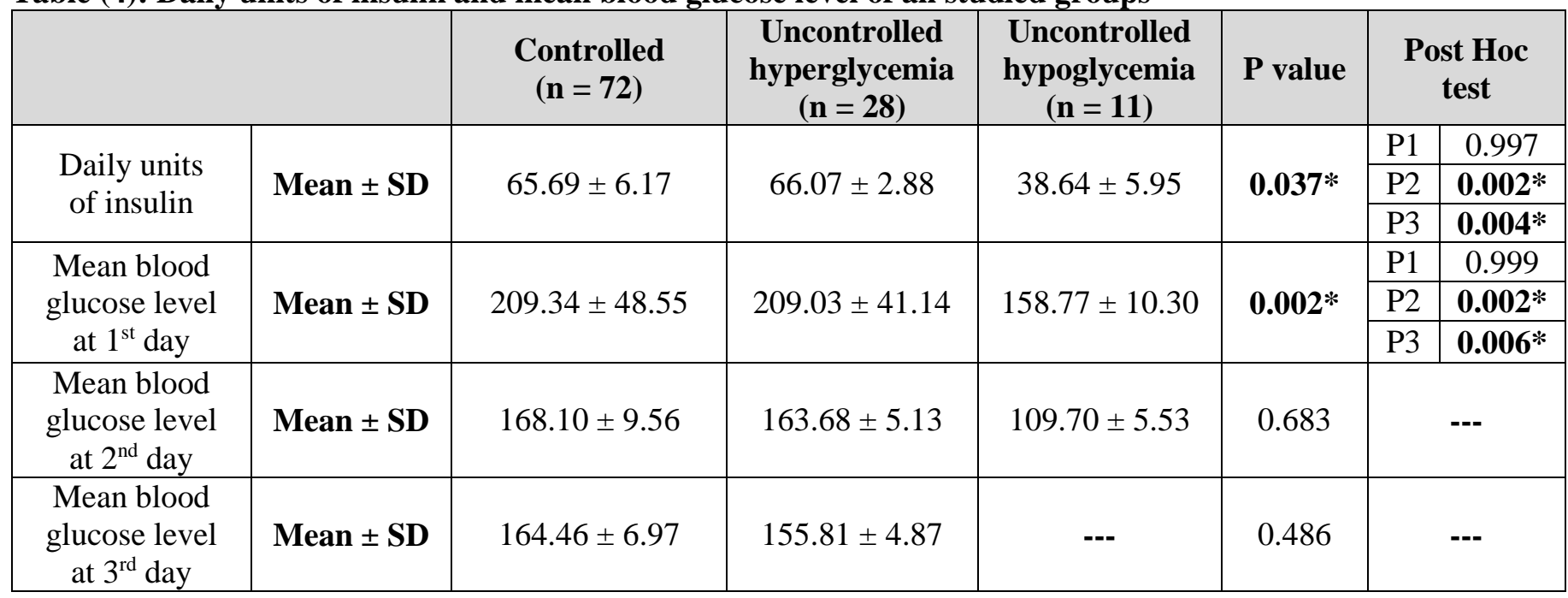

*significant as P value <0.05, P1: P value between "Controlled" group and "Uncontrolled hyperglycemia" group, P2: P value between "Controlled" group and "Uncontrolled hypoglycemia" group, P3: P value between "Uncontrolled hyperglycemia" group and "Uncontrolled hypoglycemia" group.

\section{DISCUSSION}

In critical care settings, hyperglycemia, hypoglycemia and glucose fluctuation in dysglycemic individuals are all independent risk factors for mortality (13). Close glycemic control is a laborious process that adds to the strain and expenses of nursing care ${ }^{(\mathbf{1 4})}$. In intensive care units (ICUs), intravenous (IV) shortacting regular insulin infusions are the recommended method for managing serum glucose regardless of the debate over establishing a goal for blood glucose (BG) levels. Long-acting insulin formulations, on the other hand, are seldom explored due to the drug's changed pharmacokinetic and pharmacodynamic features (15). Insulin glargine has a lower peak impact and takes around 2 hours to start working. Despite the fact that the risk of hypoglycemia is reduced in comparison to other types of long-acting insulins, it is not zero. When compared to other types of insulin, insulin glargine with intravenous insulin may be advantageous for hyperglycemic patients in critical care because of its constant plasma level and lengthy half-life ${ }^{(16)}$.

Our results were nearly agreed with that of Doshi et al. (16) who compared the effects of IV insulin (standard) with combination of IV insulin and insulin glargine (experimental). They found that using glargine with an insulin infusion leads to well-controlled Random blood sugar (RBS) during the management of acute DKA.

In accordance with our results, Shou et al. ${ }^{(17)}$ run an open-label, parallel-group trial. Patients with type 2 diabetes mellitus (T2DM) and glycated hemoglobin $(\mathrm{HbAlc}) \geq 9 \%(75 \mathrm{mmol} / \mathrm{mol})$ or showed fasting plasma glucose $(\mathrm{FPG}) \geq 11.1 \mathrm{mmol} / \mathrm{L}$ were randomly allocated to continuous subcutaneous insulin infusion (CSII) or insulin glargine with metformin and gliclazide in combination. They showed that in adults with T2DM, short-term insulin glargine and oral hypoglycemic agents (OHAs) might be a substitute to CSII for first intensive management.

In accordance with our results, Hsia et al. ${ }^{(18)}$ in a prospective randomized trial assessed 61 diabetic cases who were getting IV insulin treatment. Within 12 hours after initiating IV insulin infusion, subjects received daily injections of subcutaneous (SC) glargine $(0.25$ $\mathrm{U} / \mathrm{kg}$ body weight) in the intervention group. Up to 12 hours after the insulin infusion was stopped, capillary blood glucose readings were recoded. They showed that SC insulin glargine once-daily infusions along with IV insulin infusions are a safe way to avoid future rebound hyperglycemia while reducing the risk of hypoglycemia.

In agreement with our results, Ramos et al. (19) examined 7 enteral nutrition patients and 14 total parenteral nutrition patients were examined after switching from IV infusion insulin to SC insulin. Extrapolating from the preceding 12 hours, the first SC insulin dosage was predicted to be $50 \%$ of the daily required IV insulin. They found that with SC insulin administration, mean BG levels were $136 \pm 35 \mathrm{mg} / \mathrm{dl}$ in the enteral nutrition group and $157 \pm 37 \mathrm{mg} / \mathrm{dl}$ in the total parenteral nutrition group $(\mathrm{p}=0.01)$. For patients on enteral nutrition, an initial insulin glargine dosage of $50 \%$ of daily required IV insulin was sufficient during the switch from IV to SC insulin treatment.

In agreement with our results, Porcellati et al. (20) evaluated 24 type 1 diabetic patients following a 2week therapy by either detemir or glargine once daily to determine their pharmacokinetics (PK) and pharmacodynamics (PD). They found that glargine kept 
plasma glucose level at $103 \pm 3.6 \mathrm{mg} / \mathrm{dl}$ for up to 24 hours, and that all individuals finished the trial.

In accordance with our results, Nader $\boldsymbol{e t}$ al. (21) evaluated 110 patients who, besides daily insulin infusions, were randomly assigned to receive adjuvant placebo (control) or insulin glargine $15 \mathrm{IU} /$ day (glargine) to keep BG levels at a range of 140-180 $\mathrm{mg} / \mathrm{dl}$. They found that the average daily glucose level in the glargine group was significantly lower than in the control group, and they concluded that adding insulin glargine to regular protocols successfully lowers BG levels and minimizes the occurrence of hyperglycemia in addition to the need to regular insulin. This modification might be linked to shorter ICU stays or more hypoglycemia incidents.

In agreement with our results, Monami et al. ${ }^{(22)}$ evaluated 285 randomized controlled studies at with a length of more than 12 weeks that compared long-acting insulin analogues (glargine or detemir) to Neutral Protamine Hagedorn (NPH) insulin in type $1 \mathrm{DM}$ patients, researchers looked. They found that switching from NPH to long-acting analogues in type $1 \mathrm{DM}$ patients improved glucose control and decreased the likelihood of nocturnal and severe hypoglycemia.

In agreement with our results, Candido et al. (23) evaluated management of patients with T2DM by insulin glargine plus insulin lispro and indicated the safety and efficacy of these formulations. With insulin lispro and glargine providing comparable levels of safety and efficacy in pediatric and adult participants with type $1 \mathrm{DM}$, as well as in adult participants and those over 65 years old with T2DM. In individuals with type 1 or $2 \mathrm{DM}$ in addition to other comorbidities, these insulin formulations seemed to be safe and effective in managing T2DM.

Our results were in line with Becker $\boldsymbol{e t ~ a l . ~}{ }^{(24)}$ who compared PD and PK of a novel insulin glargine with 300 units ml(-1) (Gla-300) to insulin glargine with 100 units ml(-1) (Gla-100) in steady state in persons with type $1 \mathrm{DM}$. They showed that glargine is effective in glycemic control, and that Gla-300 had more consistent and steadier PD and PK profiles and a longer duration of action than Gla-100, allowing blood glucose control to last longer than 24 hours.

In contrast with our results, Janež et al. (25) evaluated a daily course of multiple basal/bolus insulin injections in those not fulfilling their glycemic targets or experiencing frequent or severe hypoglycemia. They found that rapid-acting prandial insulin infusion analogues were as effective as conventional human insulin and had a decreased risk of hypoglycemia.

In contrary to our results, Gao et al. ${ }^{(26)}$ evaluated, for 12 weeks, 200 patients with T2DM who were included in the trial and were randomly distributed to CSII $(n=100)$ group or Multiple daily doses of insulin (MDI) ( $\mathrm{n}=100$; before each meal and glargine before sleep) group. The individuals were placed on a continuous glucose monitoring device for 2 to 3 days during the final week of each course. They found no statistically significant variations in haemoglobin A1c (HbA1c) levels or incidence of hypoglycemia between the two groups, and that all patients in the MDI and CSII groups had satisfactory glycemic control. The MDI group's basal insulin dose was significantly lower when compared to the CSII group (P0.001).

In contrast to our results, Rungsun et al. (27) conducted a prospective noninferiority study on patients in critical care who developed hyperglycemia and required regular insulin infusion by the Intensive Care Unit glycemic control protocol. A 100 percent conversion dosage was used in the transition of eligible patients from daily regular insulin to single-dose subcutaneous insulin glargine injection. They showed that glycemic control in severely sick individuals may be achieved with a single-dose subcutaneous insulin glargine injection. In critical cases, a single dose of subcutaneous insulin glargine provided glycemic control equivalent to conventional intravenous regular insulin infusion.

In contrast to our results, Hirsch et al. (28) conducted a randomized, open-label crossover, multicenter trial including 100 participants. After a oneweek run-in period with aspart via CSII, 50 patients were randomly randomized to MDI treatment (aspart before each meal and glargine at night), while the other 50 individuals remained on CSII. They found that CSII treatment with insulin aspart resulted in decreased glycemic exposure without an increased risk of hypoglycemia when compared to MDI with insulin aspart and glargine, as evaluated by area under the curve.

In contrary to our results, Pitlick et al. (29) conducted a retrospective observational study including adult patients with type 1 or $2 \mathrm{DM}$ who were changed from Lantus (LGlar) to insulin glargine (BGlar) at five clinics. They showed that the findings of this retrospective analysis indicate that BGlar produced comparable glycemic outcomes in a real-world scenario when compared to LGlar, and that it would be a better alternative in a value-based health care system.

The effectiveness of numerous insulin regimens used to treat hyperglycemia in hospitalised patients, such as fast, glargine, NPH, or Premix insulin, was studied in a meta-analysis by Verçozaet al. ${ }^{(15)}$. In the control RBS, they found no change in insulin regimen in contrast to our results.

At the same dose, Schaschkow et al. (30) examined the effects of two modalities of insulin administration (single injections of long-acting insulin versus pump delivery of rapid-acting insulin). In contrast to our results, they showed that continuous insulin administration through pumps restored normoglycemia, resulting in a decrease in reactive oxygen species and macrophage infiltration in the liver and omentum. Long-acting insulin injections only regulated glucose levels for a short time, resulting in increased tissue stress and inflammation. 
In contrary to our results that shows APACHE II score was significantly higher in the "Uncontrolled hyperglycemia" group compared to the "Uncontrolled hypoglycemia" group but insignificantly different between the "Controlled" group and "Uncontrolled hyperglycemia" group and between "Uncontrolled hypoglycemia" group and "Controlled" group, Safari $\boldsymbol{e t}$ al. (31) evaluated enrolled 183 individuals with hyperglycemia who were above the age of 18 . The individuals were separated into DKA and non-DKA patients after the main examination. All patients' APACHE II scores were computed and compared to one another. They calculated cut-off points of APACHE II score, specificity, sensitivity and predictive value for DKA. Sixty-two patients were found to have DKA. There was no significant difference in APACHE II scores between two groups of patients $(P=0.597)$. Because there was no adequate cut-off point for APACHE II score, in cannot be applied in the prediction of DKA in hyperglycemic patients admitted in ED.

\section{CONCLUSION}

It could be concluded that use of $100 \%$ conversion single dose of long acting insulin glargine (Lantus®), Sanofi-Aventis, USA) for control of hyperglycemia in critically ill patients in ICU can be considered as an accepted good alternative to the classic use of continuous regular insulin infusion.

\section{REFERENCES}

1. Krinsley J (2003): Association between hyperglycemia and increased hospital mortality in a heterogeneous population of critically ill patients. Mayo Clin Proc., 78:1471-8.

2. Rau C, Wu S, Chen Y et al. (2017): Stress-Induced Hyperglycemia, but Not Diabetic Hyperglycemia, Is Associated with Higher Mortality in Patients with Isolated Moderate and Severe Traumatic Brain Injury: Analysis of a Propensity Score-Matched Population. Int J Environ Res Public Health, 14: 1340-44.

3. Krinsley J (2010): Moving closer to untangling a sweet web: hyperglycemia, diabetic status, and mortality in the critically ill. Crit Care Med., 38:295-6.

4. Khan S, Ibrahim M, Anwar H (2015): Frequency and Mortality Associated with Hyperglycemia in Critically Ill Children. J Coll Physicians Surg Pak., 25:878-81.

5. Douketis J (2002): Review: stress hyperglycemia after ischemic stroke indicates a greater risk for death in patients without diabetes. ACP J Club., 136:114-118.

6. Finfer S, Chittock D, Su S et al. (2009): Intensive versus conventional glucose control in critically ill patients. N Engl J Med., 360:1283-97.

7. Nichols R, Zawada E, Johnson T et al. (2008): Implementation of an insulin protocol using a multiplierbased calculation for determining infusion rates in critically ill patients. S D Med., 61:247-53.

8. Cavalcanti A, Silva E, Pereira A et al. (2009): A randomized controlled trial comparing a computerassisted insulin infusion protocol with a strict and a conventional protocol for glucose control in critically ill patients. J Crit Care, 24:371-8.

9. Pollex E, Moretti M, Koren G et al. (2011): Safety of insulin glargine use in pregnancy: a systematic review and meta-analysis. Ann Pharmacother., 45:9-16.

10. Schober E, Schoenle E, Van Dyk J et al. (2002): Comparative trial between insulin glargine and NPH insulin in children and adolescents with type 1 diabetes mellitus. J Pediatr Endocrinol Metab., 15:369-76.

11. Chakkarwar $P$, Manjrekar $\mathbf{N}$ (2005): Insulin glargine: a long acting insulin analog. J Postgrad Med., 51:68-71.

12. Bhurayanontachai $R$, Rattanaprapat $T$, Kongkamol C (2018): Comparison of glycemic control between continuous regular insulin infusion and single-dose subcutaneous insulin glargine injection in medical critically ill patients. Indian J Crit Care Med., 22:174179.

13. Lanspa M, Dickerson J, Morris A et al. (2014): Coefficient of glucose variation is independently associated with mortality in critically ill patients receiving intravenous insulin. Crit Care, 18: 86-71.

14. Samarkandy S, Al-Dorzi H, Tamim H et al. (2010): Nursing workload and perception about intensive insulin therapy in critically ill adult patients. Saudi Med J., 31:331-2.

15. Verçoza Viana M, Verçoza Viana L, Tavares A et al. (2017): Insulin Regimens to Treat Hyperglycemia in Hospitalized Patients on Nutritional Support: Systematic Review and Meta-Analyses. Ann Nutr Metab., 71:18394.

16. Doshi P, Potter A, De Los Santos D et al. (2015): Prospective randomized trial of insulin glargine in acute management of diabetic ketoacidosis in the emergency department: a pilot study. Acad Emerg Med., 22: 657-62.

17. Lin S, Chen M, Chen W et al. (2018): A Randomized Trial of Insulin Glargine plus Oral Hypoglycemic Agents versus Continuous Subcutaneous Insulin Infusion to Treat Newly Diagnosed Type 2 Diabetes. J Diabetes Res., 18:279-84.

18. Hsia E, Seggelke S, Gibbs J et al. (2012): Subcutaneous administration of glargine to diabetic patients receiving insulin infusion prevents rebound hyperglycemia. J Clin Endocrinol Metab., 97:3132-7.

19. Ramos A, Zapata L, Vera $P$ et al. (2017): Transition from intravenous insulin to subcutaneous long-acting insulin in critical care patients on enteral or parenteral nutrition. Endocrinol Diabetes Nutr., 64:552-56.

20. Porcellati F, Rossetti P, Busciantella N et al. (2007): Comparison of pharmacokinetics and dynamics of the long-acting insulin analogs glargine and detemir at steady state in type 1 diabetes: a double-blind, randomized, crossover study. Diabetes Care, 30:244752.

21. Nader N, Hamishehkar H, Naghizadeh A et al. (2020): Effect of Adding Insulin Glargine on Glycemic Control in Critically Ill Patients Admitted to Intensive Care Units: A Prospective Randomized Controlled Study. Diabetes Metab Syndr Obes., 13:671-8.

22. Monami M, Marchionni N, Mannucci E (2009): Longacting insulin analogues vs. NPH human insulin in type 1 diabetes. A meta-analysis. Diabetes Obes Metab., 11:372-8.

23. Candido R, Wyne K, Romoli E (2018): A Review of Basal-Bolus Therapy Using Insulin Glargine and Insulin Lispro in the Management of Diabetes Mellitus. Diabetes Ther., 9:927-49.

24. Becker R, Dahmen R, Bergmann K et al. (2015): New insulin glargine 300 Units $\bullet \mathrm{mL}-1$ provides a more even 
activity profile and prolonged glycemic control at steady state compared with insulin glargine 100 Units $\cdot \mathrm{mL}-1$. Diabetes Care, 38:637-43.

25. Janě̌ A, Guja C, Mitrakou A et al. (2020): Insulin Therapy in Adults with Type 1 Diabetes Mellitus: a Narrative Review. Diabetes Ther., 11:387-409.

26. Gao G, Heng X, Wang Y et al. (2014): Comparison of continuous subcutaneous insulin infusion and insulin glargine-based multiple daily insulin aspart injections with preferential adjustment of basal insulin in patients with type 2 diabetes. Exp Ther Med., 8(4):1191-6.

27. Bhurayanontachai $R$, Rattanaprapat $T$, Kongkamol C (2018): Comparison of Glycemic Control between Continuous Regular Insulin Infusion and Single-dose Subcutaneous Insulin Glargine Injection in Medical Critically Ill Patients. Indian J Crit Care Med., 22:174-9.

28. Hirsch I, Bode B, Garg S et al. (2005): Continuous subcutaneous insulin infusion (CSII) of insulin aspart versus multiple daily injection of insulin aspart/insulin glargine in type 1 diabetic patients previously treated with CSII. Diabetes Care, 28:533-8.

29. Pitlick J, Bryant G, Daly M et al. (2020): Real-World Evaluation of Dosing in Patients Converted From Insulin Glargine (Lantus) to Insulin Glargine (Basaglar). Ann Pharmacother., 54:846-51.

30. Schaschkow A, Mura C, Dal S et al. (2016): Impact of the Type of Continuous Insulin Administration on Metabolism in a Diabetic Rat Model. J Diabetes Res., 2016:8310516.

31. Safari S, Rahmani F, Soleimanpour H et al. (2014): Can APACHE II Score Predict Diabetic Ketoacidosis in Hyperglycemic Patients Presenting to Emergency Department? Anesth Pain Med., 4: 21365-21369. 